\title{
ANÁLISE DA ALIMENTAÇÃO COMPLEMENTAR NO BEBÊ A PARTIR DOS SEIS MESES DE IDADE
}

\author{
Vanessa Félix Feitosa \\ Faculdades Integradas de Patos \\ vanessatfelixf@gmail.com \\ Ana Talita da Cunha Ramos \\ Faculdades Integradas de Patos \\ ana.talita24@gmail.com
}

\author{
Ana Emilia Nascimento \\ Universidade Federal de Campina Grande \\ nemilia80@gmail.com \\ Janilson Avelino da Silva \\ Universidade Federal da Paraíba \\ janilsonsilva@globomail.com
}

\section{Resumo}

É definida como complementação alimentar o período no qual outros alimentos, sejam eles sólidos ou líquidos, são oferecidos a criança em adição ao leite materno a partir dos seis meses de idade, entretanto existem algumas controvérsias.O presente estudo tem como objetivo revisar e analisar o tempo de iniciação correta, dissertando também sobre algumas características importantes da alimentação complementar em bebês por meio de uma revisão integrativa. Foram incluídos estudos que apresentassem temática sobre a alimentação de bebês de zero aos vinte e quatro meses de idade, trabalhos em português e em inglês, estudos publicados entre 2011 e 2016, utilizando o descritor "alimentação complementar" e "complementary feeding". As bases de dados utilizadas foram: Medline/PubMed, Scielo e Lilacs. Foram encontrados 23 artigos utilizando os critérios adotados. Todos os estudos analisados mostram a importância da alimentação complementar de forma correta, iniciada a tempo que atendam as necessidades energéticas do bebê, destacando também os riscos da iniciação precoce.

Palavras-chave: Alimentação Complementar. Nutrição do Lactente.Desenvolvimento infantil.

\section{ANALYSIS OF COMPLEMENTARY FEEDING IN BABY FROM THE SIX MONTHS OF AGE}

\section{Abstract}

It is defined as complementary feeding period which other foods, whatever solid or liquid, are offered the child in addition to breast milk from six months old. This study aim to review and analyze the correct initiation of complementary feeding for babies from 6 months old. We chose to perform an integrative review. Studies were included to present theme about babies feed to the six twenty-four months of age, articles in Portuguese and English published between 2011 and 2016, only review articles, human studies using the terms "alimentação complementar" and "complementary feeding". The databases used were: Medline / PubMed(Via National Library of Medicine), SciELO (Scientific Electronic Library Online), Lilacs (Latin American and Caribbean Health Sciences). Were found 23 articles. All studies analyzed showed the importance of complementary feeding correctly, started in time to meet the energy needs of the baby, also highlighting the risks of early initiation.

Key words: Complementary feeding. Child Development. Integrative review.

\section{ANÁLISIS COMPLEMENTARIO DE POTENCIA CON BEBÉS SEIS MESES DE EDAD}

\footnotetext{
Resumen

Complemento alimenticio se define como el período en el que otros alimentos, sólidos o líquidos, se proporcionan además de la leche materna niño de seis meses idade.O presente estudio tiene como objetivo revisar y analizar la iniciación correcta de la alimentación complementaria en lactantes mayores de 6 meses. Elegimos para llevar a cabo una revisión integrativa.Foram incluyó estudios para presentar el tema acerca de los bebés se alimentan a las seis de veinte-cuarto mes de vida, el trabajo en portugués y en Inglés, los estudios publicados entre 2011 y 2016, utilizando "alimentación complementaria" y "alimentación complementaria". Las bases de datos usadas fueron: Medline / PubMed, SciELO y lilas. 23 artículos usando los criterios adoptados. Todos los estudios analizados muestran la importancia de la alimentación complementaria correctamente, comenzó a tiempo para satisfacer las necesidades energéticas del bebé, destacando también los riesgos de inicio temprano.

Palabras clave: Alimentación complementaria. Nutrición infantil. El desarrollo del niño.
} 
Análise da alimentação complementar no bebê a partir dos seis meses de idade

\section{INTRODUÇÃO}

É definida como complementação alimentar o período no qual outros alimentos, sejam eles sólidos ou líquidos, são oferecidos a criança em adição ao leite materno a partir dos seis meses de idade (WHO, 1991). Há uma preocupação muito importante a respeito da qualidade dos alimentos que estão sendo ofertados na infância. Os alimentos processados devem ser evitados por possuírem em sua composição sódio em excesso, muitos conservantes e açúcares, podendo causar uma série de patologias como diabetes, hipertensão arterial e obesidade (BRASIL, 2012).

O leite humano oferece os nutrientes que a criança necessita para iniciar uma vida saudável e representa o alimento essencial para o lactente até o sexto mês de vida como alimento exclusivo, a partir de então deve ser complementado com outras fontes nutricionais até os dois anos de idade (BRASIL, 2010).

As orientações sobre introdução de alimentos para os bebês merecem minuciosa atenção e engajamento por parte dos profissionais de sáude e da família, assim sendo, o nutricionista tem fundamental importância fornecendo uma orientação adequada aos pais sobre a introdução de alimentos corretos na dieta das crianças, assim proporcionando meios para que elas alcancem seu potencial biológico e cresçam com maior qualidade de vida. Objetiva-se assim a melhor nutrição possível para a demanda de um corpo que ainda está em desenvolvimento e é imaturo (CARVALHO; OLIVEIRA; SANTOS, 2010).

A alimentação saudável a partir dos 6 meses de idade é também muito importante, pois através dela a criança terá energia suficiente para realizar as suas atividades e se desenvolver com menores chances de adquirir doenças ou problemas de cunho nutricional (BRASIL, 2010).

A partir dos 6 meses de idade o leite materno deixa de ser suficiente para satisfazer todas as necessidades do organismo do bebê, a partir de então ele deve estar preparado para receber os alimentos e uma nova fase será iniciada. Os alimentos serão oferecidos em complementação ao leite materno e não como substitutos. Entretanto, sabe-se que existe uma elevada prevalência de mães que começam a realizar a introdução de outros alimentos em substituição ao leite materno, podendo trazer alguns prejuízos para a criança (SCHINCAGLIA et al., 2015). Diante desses aspectos, qual o tempo correto para a introdução dos alimentos complementares para bebês? 
Análise da alimentação complementar no bebê a partir dos seis meses de idade

O presente estudo tem como objetivo revisar e analisar o tempo de iniciação correta da alimentação complementar, dissertando também sobre algumas características importantes dessa fase em bebês por meio de uma revisão integrativa.

\section{METODOLOGIA}

Escolheu-se realizar uma revisão integrativa, pois permite realizar uma relação entre a pesquisa científica e o âmbito da atuação profissional. Além disso, contribui para o aprofundamento do conhecimento do tema investigado (MENDES; SILVEIRA; GALVÃO, 2008).

Foram incluídos estudos que apresentassem temática sobre a alimentação complementar de bebês de zero aos vinte e quatro meses de idade, trabalhos em português e em inglês, publicados entre 2011 e 2016, utilizando o descritor "alimentação complementar" e o seu correlato em inglês "complementary feeding".

As bases de dados utilizadas foram: Medline/PubMed (Via National Library of Medicine), Scielo (Scientific Eletronic Library Online) e Lilacs (Literatura Latino Americana e do Caribe em Ciências da Saúde). Foram escolhidas por serem bases de dados da literatura nacional e internacional em Ciências da Saúde.

Após a avaliação e leitura dos papers, estes foram sistematizados em uma tabela, com referência, descrição do título do trabalho e tempo de alimentação, de acordo com a disponibilidade dessa informação : 
Análise da alimentação complementar no bebê a partir dos seis meses de idade

Tabela 1 - Resumo dos estudos encontrados na presente investigação sobre o tempo correto da introdução de alimentos complementares nos bebês.

\begin{tabular}{|c|c|c|}
\hline REFERÊNCIA & TÍTULO & $\begin{array}{l}\text { TEMPO DE } \\
\text { INICIAÇÃO }\end{array}$ \\
\hline Lima et al. ${ }^{11}$ & $\begin{array}{l}\text { Práticas alimentares no primeiro ano de } \\
\text { vida }\end{array}$ & - \\
\hline Martins et al. ${ }^{12}$ & $\begin{array}{l}\text { Conhecimentos maternos: influência na } \\
\text { introdução da alimentação complementar }\end{array}$ & - \\
\hline Campos et al. ${ }^{10}$ & $\begin{array}{l}\text { Aconselhamento nutricional de crianças } \\
\text { menores de dois anos de idade: } \\
\text { potencialidades e obstáculos como } \\
\text { desafios estratégicos }\end{array}$ & - \\
\hline Oliveira et al. $^{7}$ & $\begin{array}{l}\text { Padrões de alimentação complementar } \\
\text { no primeiro ano de vida na cidade do Rio } \\
\text { de Janeiro, Brasil: tendências temporais } \\
\text { de } 1998 \text { a } 2008\end{array}$ & $\begin{array}{l}56,3 \% \text { das crianças estudadas } \\
\text { receberam algum tipo de } \\
\text { alimento antes dos } 6 \text { meses }\end{array}$ \\
\hline Souza et al. ${ }^{8}$ & $\begin{array}{l}\text { Alimentação complementar de lactentes } \\
\text { no primeiro ano de vida: ênfase nas papas } \\
\text { principais }\end{array}$ & $\begin{array}{c}52,4 \% \text { das crianças } \\
\text { receberam alimentos antes } \\
\text { dos } 6 \text { meses }\end{array}$ \\
\hline Alvisi et al. ${ }^{9}$ & $\begin{array}{l}\text { Recomendações sobre alimentação } \\
\text { complementar para crianças saudáveis, a } \\
\text { termo }\end{array}$ & $\begin{array}{c}90 \% \text { das crianças } \\
\text { acompanhadas receberam } \\
\text { alimentos sólidos antes dos } 6 \\
\text { meses }\end{array}$ \\
\hline
\end{tabular}

\section{RESULTADOS E DISCUSSÃO}

Foram encontrados 23 artigos. Tendo uma amostra de 6 artigos (Tabela 1), em que o tema em análise relacionava-se com os estudos encontrados. Os estudos excluídos da análise não atendiam aos critérios de elegibilidade, relacionando a alimentação complementar com outros fatores que não tinha relevância para a presente pesquisa. Os estudos analisados mostram a importância da alimentação complementar de forma correta, iniciada a tempo que atendam as necessidades energéticas do bebê, destacando também os riscos da iniciação precoce. A introdução deve ocorrer a partir dos 6 meses, de forma lenta e gradual, ofertando alimentos nutritivos, sólidos ou líquidos, diferente do leite materno, respeitando sempre o crescimento, apetite da criança e capacidade de mastigação. O Ministério da Saúde recomenda que seja iniciada respeitando os meses da criança e horários de cada refeição (BRASIL, 2013). 
Análise da alimentação complementar no bebê a partir dos seis meses de idade

Segundo Oliveira et al. (2014) e Souza et al. (2014), que tiveram seus estudos realizados com bebês analisando o tempo da iniciação da alimentação, pode-se observar que as crianças acompanhadas, cerca de 56,3\% e 52,4\% respectivamente, tiveram uma iniciação de forma precoce, a partir dos 4 meses, sendo assim introduzido na alimentação: chás, água ou outro tipo de leite associado ao leite materno, tendo ênfase no leite de vaca integral. O leite de vaca integral está associado com o desenvolvimento de doenças, a curto e longo prazo, podendo acarretar doenças crônicas na idade adulta. Corroborando assim com o estudo de Alvisiet al. (2015), que no acompanhamento das crianças estudadas identificou que $90 \%$ dos bebês tiveram a introdução de algum tipo de alimento sólido na alimentação antes do $6^{\circ}$ mês de vida.

Um dos fatores mais comuns para introdução precoce adotada pelas mães ou cuidadoras desses bebês está ligado ao retorno da mãe ao trabalho, como também as crenças, mitos e costumes adotados por algumas dessas mulheres. Destacando assim, a importância da orientação dessas mães e/ou cuidadoras, acolhendo-as e respeitando-as em suas particularidades, dificuldades, conhecimentos prévios, êxitos e satisfações (CAMPOS; COTA; ROSANGELA, 2014).

Todos os estudos indicam que a introdução precoce de alimentos traz desvantagens para o lactente e podem comprometer a sua saúde. Verificou-se que pode ocorrer a redução no tempo de amamentação exclusiva, aumentando o risco de reações alérgicas. Por outro lado, quando a alimentação complementar é introduzida tardiamente, pode retardar o crescimento da criança, aumentando o risco de deficiências de micronutrientes essenciais como ferro, cálcio, ácido fólico, vitamina A, vitamina C e zinco (BRASIL, 2013).

De acordo com Lima et al (2011), citado por Martins et al (2012), a prática de uma alimentação precoce e iniciada de forma incorreta pode estar ligada ligada à existência de crenças e práticas da cultura brasileira mostrando-se conflitivas com as recomendações para alimentação do lactente. Os cuidados maternos representam forte impacto sobre a saúde da criança e assim tendo influencia na formação dos hábitos alimentares. Normalmente, mães com hábitos alimentares inadequados dificilmente irão estabelecer uma alimentação infantil adequada.

A prática de uma dieta de transição alimentar inadequada têm mostrado uma imagem de alimentação quantitativamente e qualitativamente imprópria, destacando o grando risco de causar sérios problemas nutricionais em idades posteriores, como anemia e deficiência de vitamina A ou o consumo excessivo de nutrientes, acarretando no desenvolvimento de doenças crônicas não transmissíveis (SOUZA, 2014). 
Análise da alimentação complementar no bebê a partir dos seis meses de idade

A importância do profissional de nutrição juntamente com os demais profissionais de sáude, principalmente os ligados a atenção básica, para orientação e conscientização das mães e/ou cuidadoras, em relação a forma correta de alimentar o bebê, respeitando cada fase, visando sempre uma boa saúde e o bem estar é de fundamental necessidade. Oferecendo a criança todos os grupos de alimentos tais como, cereais, tubérculos, pães, verduras, legumes, frutas, leite e produtos lácteos, (3 porções diárias de cada grupo) carnes, míudos, ovos e leguminosas (2 porções diárias de cada grupo) e os açúcares só são recomendado a partir dos 2 anos de idade (ALVISI; BRUSA; ALBORESI, 2015).

Ofertando sempre uma refeição que esteja nutricionalmente equilibrada, harmoniosa, de forma adequada o que significa que devem fornecer energia, macro e micronutrientes que satisfaçam às necessidades nutricionais de uma criança em crescimento; sejam seguros do ponto de vista higiênico-sanitário e sejam adequadamente ministrados, ofertados de acordo com os sinais de apetite e saciedade da criança, essas práticas asseguram o bom desenvolvimento físico, neurológico e motor da criança (MARTINS; HAACK, 2012).

Todos os hábitos alimentares buscam um futuro bem próximo de qualidade de vida e saúde. As práticas adotadas nos primeiros vinte e quatro meses de vida é de extrema importância, pois é o período no qual os hábitos alimentares são estabelecidos e consequentemente se refletirão na adolescência e na idade adulta (OLIVEIRA; CASTRO, 2014; LIMA et al, 2011).

\section{CONSIDERAÇÕES FINAIS}

A realização de uma revisão integrativa sobre práticas alimentares nos primeiros meses de vida constatou a importância tanto da escolha dos alimentos a serem ofertados, quanto ao tempo em que está sendo introduzido, devido aos diversos benéficios para o lactente, contribuindo com os seus futuros hábitos alimentares.

Os estudos constataram que ainda há uma grande dificuldade em relação a essa iniciação alimentar de forma correta, devido as crenças, tabus e costumes familiares, interferindo assim de forma direta nas escolhas dos alimentos ofertados. Nota-se também que a introdução alimentar inadequada traz importantes consequências na saúde da criança a curto e a longo prazo, podendo acarretar doenças crônicas na idade adulta.

Observa-se que há uma grande lacuna a ser preenchida em relação aos profissionais de sáude sobre a temática estudada, ressaltando a importância de profissional 
Análise da alimentação complementar no bebê a partir dos seis meses de idade

apto a prescrever e orientar sobre uma alimentação adequada de acordo com as necessidades de cada individuo que é o nutricionista.

\section{REFERÊNCIAS}

ALVISI, Patrizia; BRUSA, Sandra; ALBORESI, Stefano; et al., Recommendations on complementary feeding for healthy, full-term infants.Ital J Pediatr. 2015; 41: 36.

BRASIL. Ministério da Saúde. Dez passos para uma alimentação saudável Guia alimentar para crianças menores de dois anos. Um guia para o profissional da saúde na atenção básica. $2^{\mathrm{a}}$ ed. Brasília-DF. 2012.

BRASIL. Ministério da Saúde. Dez passos para uma alimentação saudável: Guia alimentar para crianças menores de dois anos. Brasília-DF. 2010.

BRASIL. Ministério da Saúde. Dez passos para uma alimentação saudável: guia alimentar para crianças menores de dois anos: um guia para o profissional da saúde na atenção básica - 2 ed. - Brasília: Ministério da Saúde, 2013.

CAMPOS, Aline Aparecida de Oliveira; COTTA, Rosângela Minardi Mitre; OLIVEIRA, Julicristie Machado; et al., Aconselhamento nutricional de crianças menores de dois anos de idade: potencialidades e obstáculos como desafios estratégicos. Ciênc. saúde coletiva, Fev 2014, vol.19, no.2, p.529-538. ISSN 1413-8123. Disponível em: <http://www.scielo.br/scielo.php?script=sci_arttext\&pid=S1413-81232014000200529>

CARVALHO, Adriana Palhares de; OLIVEIRA, Verena Bartkowiak de; SANTOS, Luana Caroline dos. Hábitos alimentares e práticas de educação nutricional: atenção a crianças de escola de Belo Horizonte-MG. Jornal de Pediatria. 2010; 32(1):20-27.

LIMA, Ana Paula Esmeraldo; JAVORSKI, Marly; VASCONCELOS, Maria Gorete Lucena. Práticas alimentares no primeiro ano de vida. Revista Brasileira de Enfermagem.vol.64 no.5 Brasília Sept./Oct. $2011 . \quad$ Disponível em: <http://www.scielo.br/scielo.php?script=sci_arttext\&pid=S0034-71672011000500017>

MARTINS, Murielle de Lucena; HAACK, Adriana. Conhecimentos maternos: influência na introdução da Alimentação complementar. Ciências Saúde. 2012; 23(3):263-270. Disponível

em: <http://dominioprovisorio.tempsite.ws/pesquisa/revista/2012Vol23_3_8_Conhecime ntosMaternosInfluencia.pdf $>$

MENDES, Karina Dal Sasso; SILVEIRA, Renata Cristina de Campos Pereira; GALVAO, Cristina Maria. Revisão integrativa: método de pesquisa para a incorporação de evidências na saúde e na enfermagem. Texto contexto - enferm., Florianópolis, v. 17, n. 4, Dec. 2008. Disponível em <http://dx.doi.org/10.1590/S0104-07072008000400018>.

OLIVEIRA, Daniel Alves ; CASTRO, Inês Rugani Ribeiro de; JAIME, Patricia Constante. Padrões de alimentação complementar no primeiro ano de vida no Município do Rio de Janeiro, Brasil: tendência temporal (1998-2008). Cad. Saúde 
Análise da alimentação complementar no bebê a partir dos seis meses de idade

Pública. 2014, vol.30, n.8, pp.1755-1764. ISSN 0102-311X. Disponível em: <http://www.scielo.br/scielo.php?pid=S0102311X2014000801755\&script=sci_abstract\&t $\operatorname{lng}=\mathrm{pt}>$

SCHINCAGLIA et al. Práticas alimentares e fatores associados à introduçãoprecoce da alimentação complementar entre crianças menores de seis meses na região noroeste de Goiânia. Epidemiol. Serv. Saúde,v.24, n.3, p.465-474, 2015.

SOUZA, Fabíola Isabel Suano de et al. Alimentação complementar de lactentes no primeiro ano de vida: ênfase nas papas principais. Rev. Assoc. Med. Bras. 2014, vol.60, n.3, pp.231-235. ISSN 0104-4230. Disponível em: <http://www.scielo.br/scielo.php?pid=S010442302014000300231\&script=sci_abstract\&tl $\mathrm{ng}=\mathrm{pt}>$

WHO. Division of Child Health and Development, World Health Organization. Indicators for assessing breastfeeding practices. Geneva, 1991. 